\title{
Health-related quality of life of patients with HIV/AIDS at a tertiary care teaching hospital in Ethiopia
}

Yared Belete Belay ${ }^{{ }^{*}}$ (D), Eskinder Eshetu Ali² , Beate Sander ${ }^{3,4,5,6}$ and Gebremedhin Beedemariam Gebretekle $2,3,4$

\begin{abstract}
Background: Patients' health-related quality of life (HRQoL) and health state utility values are critical inputs in the clinical and economic evaluation of treatments for human immunodeficiency virus (HIV)/acquired immunodeficiency syndrome (AIDS). However, information on health state utility values is lacking in the context of Ethiopia. Here, we aimed to assess HRQoL and determine health state utility values and factors that influence the values among HIV/ AIDS patients in Ethiopia.
\end{abstract}

Methods: A cross-sectional study was conducted among 511 HIV/AIDS patients at Tikur Anbessa Specialized Hospital in Ethiopia. Patients aged 18 years or older were eligible for the interview and those who were mentally unstable and with hearing impairment were excluded from the study. We performed face-to-face interviews using EuroQol-5 Dimensions-5 Levels (EQ-5D-5L) in combination with EuroQol-Visual Analog Scales (EQ-VAS). Level-specific disutility coefficients obtained from the general population were used for computing utility values. Patients' health profiles were described using percentages and different statistical analysis were conducted to determine factors associated with the EQ-5D index and EQ-VAS scores.

Results: A total of 511 patients participated in the study. A higher proportion of patients reported slight or more severe problems on the anxiety/depression (55.2\%) and pain/discomfort (51.3\%) dimensions. The overall median utility value of HIV/AIDS patients was 0.94 (IQR $=0.87,1)$ from the $E Q-5 D$ index and $80 \%(I Q R=70 \%, 90 \%)$ from the EQ-VAS scores. Demographic characteristics including age, occupational status, and household monthly income significantly affected patient's utility values. Moreover, statistically significant $(p<0.001)$ differences were seen between the EQ-5D index values of patients with different CD4 count intervals. Furthermore, number of medicines that the patients were taking at the time of the study and comorbidities were significantly associated with the EQ-5D utility index and EQ-VAS score, $p<0.001$.

Conclusions: The anxiety/depression and pain/ discomfort dimensions were identified to have critical influence in reducing the HRQoL of adult HIV/AIDS patients in the context of Ethiopia. The study is also the first to use the EQ5D-5L tool to identify health state utility values for Ethiopian adult HIV/AIDS patients. Future economic evaluations of HIV/AIDS interventions are encouraged to employ the identified utility values.

Keywords: EQ-5D-5L, HIV/AIDS, Quality of life, Utility, EuroQol

*Correspondence: yared.belete@mu.edu.et

' School of Pharmacy, College of Health Sciences, Mekelle University, Mek'ele, Ethiopia

Full list of author information is available at the end of the article

\section{Introduction}

Human immunodeficiency virus (HIV)/acquired immunodeficiency syndrome (AIDS) is a major issue of public health relevance globally. The health and economic burden of the disease is even more daunting in low-income adaptation, distribution and reproduction in any medium or format, as long as you give appropriate credit to the original author(s) and the source, provide a link to the Creative Commons licence, and indicate if changes were made. The images or other third party material in this article are included in the article's Creative Commons licence, unless indicated otherwise in a credit line to the material. If material is not included in the article's Creative Commons licence and your intended use is not permitted by statutory regulation or exceeds the permitted use, you will need to obtain permission directly from the copyright holder. To view a copy of this licence, visit http://creativecommons.org/licenses/by/4.0/. The Creative Commons Public Domain Dedication waiver (http://creativecommons.org/publicdomain/zero/1.0/) applies to the data made available in this article, unless otherwise stated in a credit line to the data. 
countries, which include most African nations [1-3]. Ethiopia is not an exception and HIV/AIDS is a significant health concern in the country. With a one percent annual prevalence, the disease accounts for $19 \%$ of Ethiopia's national health expenditure, despite significant improvements as a result of increased access to antiretroviral treatment (ART) $[4,5]$.

Although significant progress has been made in drug discovery for the treatment of HIV/AIDS, the currently available medicines fall short of curing the disease. The lack of curative therapy impacts patients' health-related quality of life (HRQoL) and treatment outcomes $[6,7]$. Hence, health-related disability and disfunction should be assessed using HRQoL. The health state utility value which is a numerical description of HRQoL can be used to express HRQoL and it incorporates the preference of people. Both the health state utility and HRQoL helps to assess the need for and outcome of interventions from the experience of patients. Health state utility value is the preferred parameter used in health technology assessment $[8,9]$. Several tools have been developed and validated for measuring HRQoL in patients including WHOQOL-HIV instrument and EQ-5D [10-12]. The capacity of these tools range from simple measurement of symptom burdens (such as the World Health Organization Quality of Life in HIV (WHOQOL-HIV) instrument) to the quantification of health state utility values. In this regard, the EuroQol-5 dimensions (EQ-5D) tool is significant because of its ability to generate utility scores, which are critical in full-fledged health economic evaluations involving cost utility analysis $[10,13]$. In such analyses, the utility is used as a good metric for comparison of quality-adjusted life-year between alternative interventions [14-16].

In Ethiopia, several studies have assessed HRQoL of HIV/AIDS patients using the WHOQOL-HIV instrument [17-20]. However, there is a paucity of studies that employ tools such as the EQ-5D that incorporate societal preference and provide effective means to assess impact of the disease across different health states [14]. As a result, health care decision-makers in Ethiopia still lack well-established utility values for HIV/AIDS patients based on different health states of the disease. To this effect, the current study aimed to assess HRQoL and determine health state utility values and factors that influence the values among HIV/AIDS patients in Ethiopia.

\section{Methods}

\section{Study design and setting}

An institution-based cross-sectional study was conducted from April to July 2019 at Tikur Anbessa Specialized Hospital (TASH), the largest tertiary teaching hospital (affiliated with Addis Ababa University) in Ethiopia. The hospital has 1,204 health professionals and 800 beds serving more than 500,000 patients per year. The ART clinic pharmacy is one of the 12 pharmacy departments within the hospital and approximately 4,000 patients are served annually in this pharmacy.

\section{Recruitment of participants}

All patients visiting the ART pharmacy at TASH were considered source population while patients visiting the ART pharmacy of the hospital during the data collection period were the study population. Participants had to be 18 years or older to be eligible for the interview. In contrast, patients with incomplete medical chart records (absence of CD4 data), those who were mentally unstable and with hearing impairment were excluded from the study.

The total number of participants was estimated based on a single proportion formula [21], considering a Z-value of 1.96 with a $95 \%$ level of confidence and $4 \%$ of a margin of error. The proportion (P) for sample size estimation was taken from a previous study which assessed the quality of life of HIV/AIDS patients at university hospital in Ethiopia and reported that $~ 33 \%$ of patients rated their overall perceived HRQoL as good [19]. According to TASH's ART pharmacy electronic dispensing tool, the total number of active ART users was 3,828 in March 2019 . With a $5 \%$ non-response rate, the final sample size was estimated to be 511 .

\section{Data collection instrument}

In this study, the Amharic versions of the 5-level EQ-5D (EQ-5D-5L) and the EuroQol-visual analog scale (EQVAS) were used to evaluate HRQoL and generate utility scores. EQ-5D is a generic, preference-based and multi-attribute utility evaluation instrument developed by the EuroQol group. It is a widely recommended tool to generate input data for economic evaluation [13]. The tool was originally developed to include three levels of severity in five dimensions (EQ-5D-3L). Since 2009, the EuroQol group introduced the 5-level EQ-5D version, which is shown to have better sensitivity and reduced ceiling effect in comparison to EQ-5D-3L. EQ-5D-5L is designed to include mobility, self-care, usual activities, pain/discomfort, and anxiety/depression dimensions. Each dimension is then measured on five levels ranging from no problem to an extremely severe problem or unable to perform any activities-VAS was used to record patient's self-rated health status on a $20-\mathrm{cm}$ vertical scale with endpoints 0 ('the worst health you can imagine') and 100 ('the best health you can imagine') [15, 22]. Prior studies involving samples from the general population showed that the Amharic versions of EQ-5D-5L and 
EQ-VAS instruments are feasible and culturally acceptable in the context of Ethiopia [23].

In addition to EQ-5D-5L and EQ-VAS, the data collection tool contained questions related to patients' sociodemographic characteristics and checklist for recording clinical characteristics from the patient medical charts. Except for the checklist, all the questions were in the Amharic language (the working language of the country), to ensure understandability by patients. Since the information in the patient chart was documented in English, using the English version of the checklist was suitable to avoid potential confusion during data collection.

\section{Data collection procedure and quality assurance}

Data collection was done by one postgraduate pharmacy student and two pharmacy staff working in the ART pharmacy who took a half-day training on the proper conduct of the study. Face-to-face interviews were carried out to collect data on socio-demographic, EQ-5D-5L and EQ-VAS questions. Information related to clinical characteristics were extracted from the medical records after completion of the interview with patients. All the collected data were checked for their completeness, accuracy and consistency by the first author (YBB) on the site of data collection. Prior to analysis, data cleaning was done by observing the descriptive statistics output.

\section{Data analysis}

The utility score was calculated using Microsoft excel. Patient health profiles from EQ-5D-5L were converted into a single index (utility) based on the preference of the general population of Ethiopia. EQ-5D-5L index and EQ-VAS scores were used to describe the HRQoL of the patients. Coefficients that represented level-specific disutility values obtained from the general population using a hybrid regression model were used for computing utility value. Four dummies were created for each dimension by considering level one as a reference to represent a decrease in utility in moving from one level to the next higher level (e.g. moving from MO1 to MO2 resulted in a utility decrement of 0.0337341 ). The 20 parameters reported from the general population for all the five health domains [23] were used to compute utility score (Eq. 1). "mo=mobility; $\quad$ "sc = self-care; "ua = usual activity; "pd = pain/discomfort; "ad=Anxiety/depression.

Equation 1. Equation for computing utility score.

Statistical analysis was performed using IBM SPSS Statistics for Windows, Version 23.0. (IBM Corp., Armonk, NY, USA). Descriptive statistics including frequency and percentage were used to describe the health profile of the study population. Kolmogorov-Smirnov, ShapiroWilk test $(p<0.001)$ and visual inspection of histogram revealed that the distribution of EQ-5D index values and EQ-VAS scores were skewed and kurtotic. Therefore, we used a non-parametric test to compare the utility value across different groups. Median and interquartile range (IQR) were used to characterize the included samples with respect to EQ-5D and EQ-VAS output. MannWhitney $U$ test was used to assess significance of difference of utilities for variables with two categories while Kruskal-Wallis test was used for comparison of utility value among variables with more than two groups. The mean rank was used to compare the difference across different groups and $p$-value $<0.05$ was considered as a cutpoint for determining statistical significance.

\section{Results}

\section{Participants' demographic and clinical characteristics}

This study enrolled a total of 511 patients with wideranging demographic and clinical characteristics. The mean age of the participants was $42 \pm 11$ years (ranged: 18 to 80 years). A total of 325 (63.6\%) of the participants were within the age range of $30-49$ years; 395 (77.3\%) never drunk alcohol and 496 (97.1\%) never smoked cigarettes (Table 1). The majority of the participants (407, $80 \%$ ) had a CD4 value of above 200 cells $/ \mathrm{mm}^{3}$ and few (22, 4.3\%) had CD4 count below 100 cells $/ \mathrm{mm}^{3}$. In contrast, $440(86 \%)$ participants had a viral load below the limit of detection (50 copies/ml). With 297 (58.1\%) of patients taking it, TDF/3TC/EFV was found to be the most frequently prescribed ART regimen (Table 2).

\section{Participants' health profile}

Overall, $92 \%, 83.4 \%$ and $80.6 \%$ of the participants reported "no problem" in the self-care, mobility and usual activity domains, respectively. In contrast, $55.2 \%$ and

$$
\begin{aligned}
\text { Utility value }= & 1-(\mathrm{mo} 2 * 0.0337341+\mathrm{mo} 3 * 0.0644715+\operatorname{mo} 4 * 0.2276493+\operatorname{mo} 5 * 0.3598963) \\
& +(\mathrm{sc} 2 * 0.0235125+\mathrm{sc} 3 * 0.0394815+\mathrm{sc} 4 * 0.1419238+\mathrm{sc} 5 * 0.2223553) \\
& +(\mathrm{ua} 2 * 0.0323013+\mathrm{ua} 3 * 0.0482993+\mathrm{ua} 4 * 0.1573934+\mathrm{ua} 5 * 0.2721253) \\
& +(\mathrm{pd} 2 * 0.0360808+\mathrm{pd} 3 * 0.0515949+\mathrm{pd} 4 * 0.2703189+\mathrm{pd} 5 * 0.4063984) \\
& +(\mathrm{ad} 2 * 0.0258862+\mathrm{ad} 3 * 0.0848133+\mathrm{ad} 4 * 0.2987388+\mathrm{ad} 5 * 0.4577938)
\end{aligned}
$$


Table 1 Socio-demographic characteristics and reported health problem of HIV/AIDS patients, TASH, Ethiopia, 2019 $(\mathrm{n}=\mathbf{5 1 1})$

\begin{tabular}{|c|c|c|c|c|c|c|}
\hline \multirow[t]{2}{*}{ Patient characteristics } & \multirow{2}{*}{$\begin{array}{l}\text { Total number } \\
\text { of participant } n(\%)\end{array}$} & \multicolumn{5}{|c|}{ Reported health problem in EQ-5D health dimensions } \\
\hline & & $\begin{array}{l}\text { Mobility } \\
\text { n (\%) }\end{array}$ & $\begin{array}{l}\text { Self-care } \\
\text { n (\%) }\end{array}$ & $\begin{array}{l}\text { Usual activity } \\
\text { n (\%) }\end{array}$ & $\begin{array}{l}\text { Pain/Discomfort } \\
\text { n (\%) }\end{array}$ & $\begin{array}{l}\text { Anxiety/Depression } \\
n(\%)\end{array}$ \\
\hline \multicolumn{7}{|l|}{ Gender } \\
\hline Male & $202(39.5)$ & $33(16.3)$ & $20(9.9)$ & $41(20.3)$ & $99(49.0)$ & $112(55.4)$ \\
\hline Female & $309(60.5)$ & $52(16.8)$ & $21(6.8)$ & $58(18.8)$ & $163(58.2)$ & $170(55.0)$ \\
\hline \multicolumn{7}{|l|}{ Age (in years) } \\
\hline $18-29$ & $63(12.3)$ & $4(6.3)$ & $3(4.8)$ & $11(17.5)$ & $38(60.3)$ & $45(71.4)$ \\
\hline 30-39 & $141(27.6)$ & $21(14.9)$ & $7(5.0)$ & $20(14.2)$ & $63(44.7)$ & 70 (49.6) \\
\hline $40-49$ & $184(36)$ & $28(15.2)$ & $13(7.1)$ & $34(18.5)$ & $94(51.1)$ & $94(51.1)$ \\
\hline $50-59$ & $77(15.1)$ & $19(24.7)$ & $9(11.7)$ & $17(22.1)$ & $34(44.2)$ & $41(53.2)$ \\
\hline$>60$ & $46(9.0)$ & $13(28.3)$ & $9(19.6)$ & $17(37.0)$ & $33(71.7)$ & $32(69.6)$ \\
\hline \multicolumn{7}{|l|}{ Marital status } \\
\hline Unmarried & $121(23.7)$ & $17(14.0)$ & $5(4.1)$ & $24(19.8)$ & $63(52.1)$ & $73(60.3)$ \\
\hline Married & $278(54.4)$ & $233(83.8)$ & $25(9.0)$ & $48(17.3)$ & $132(47.5)$ & $141(50.7)$ \\
\hline Divorced & $47(9.2)$ & $6(12.8)$ & $2(4.3)$ & $11(23,4)$ & $26(55.3)$ & $24(51.1)$ \\
\hline Widowed & $65(12.7)$ & $17(26.2)$ & $9(13.8)$ & $16(24.6)$ & $41(63.1)$ & $44(67.7)$ \\
\hline \multicolumn{7}{|l|}{ Education } \\
\hline No formal education & $61(12.0)$ & $9(14.8)$ & $5(8.2)$ & $11(18.0)$ & $39(63.9)$ & $36(59.0)$ \\
\hline Grade $(1-4)$ & $88(17.2)$ & $14(15.9)$ & $9(10.2)$ & $23(26.1)$ & $44(50.0)$ & $53(60.2)$ \\
\hline Grade (5-8) & $133(26.0)$ & $23(17.3)$ & $17(12.8)$ & $30(22.6)$ & $70(52.6)$ & $73(54.9)$ \\
\hline High school & $132(25.8)$ & $29(22.0)$ & $5(3.8)$ & $20(15.2)$ & $66(50.0)$ & $71(53.8)$ \\
\hline TVET/Diploma & $61(12.0)$ & $6(9.8)$ & $4(6.6)$ & $10(16.4)$ & $29(47.5)$ & $35(57.4)$ \\
\hline 1st degree and above & $36(7.0)$ & $4(11.1)$ & $1(2.8)$ & $5(13.9)$ & $14(38.9)$ & $14(38.9)$ \\
\hline \multicolumn{7}{|l|}{ Occupation } \\
\hline Employed & $92(18.1)$ & $17(18.5)$ & $8(8.7)$ & $11(12.0)$ & $43(46.7)$ & $45(48.9)$ \\
\hline Private business & $265(52.1)$ & $33(12.5)$ & $14(5.3)$ & 49 (18.5) & $132(49.8)$ & $142(53.6)$ \\
\hline Pensioner & $13(2.6)$ & $5(38.5)$ & $4(30.8)$ & $7(53.8)$ & $11(84.6)$ & $11(84.6)$ \\
\hline Student & $17(3.3)$ & $1(5.9)$ & - & $2(11.8)$ & $11(64.7)$ & $13(76.5)$ \\
\hline Housewife & $97(19.1)$ & 19 (19.6) & $10(10.3)$ & 19 (19.6) & $46(47.4)$ & $54(55.7)$ \\
\hline Others* & $25(4.9)$ & $9(36.0)$ & $5(20.0)$ & $10(40.0)$ & $17(68.0)$ & $15(60.0)$ \\
\hline \multicolumn{7}{|l|}{ Income } \\
\hline$<1700$ ETB & $164(33.7)$ & $34(20.7)$ & $15(9.1)$ & $38(23.2)$ & $92(56.1)$ & $95(57.9)$ \\
\hline 1700-5000 ETB & $222(45.6)$ & $33(14.9)$ & $15(6.8)$ & 44 (19.8) & $123(55.4)$ & $135(60.8)$ \\
\hline$>5000$ ETB & $101(20.7)$ & $11(10.9)$ & $8(7.9)$ & $11(10.9)$ & 38 (37.6) & $42(41.6)$ \\
\hline \multicolumn{7}{|l|}{ Smoking habit } \\
\hline Daily & $2(0.4)$ & - & - & - & - & $1(50.0)$ \\
\hline Occasionally & $13(2.5)$ & $1(7.7)$ & - & $4(30.8)$ & $8(61.5)$ & $9(69.2)$ \\
\hline Not at all & $496(97.1)$ & $84(16.9)$ & $41(8.3)$ & 95 (19.2) & $254(51.2)$ & $272(54.8)$ \\
\hline \multicolumn{7}{|l|}{ Alcohol taking habit } \\
\hline Never & $395(77.3)$ & $71(18)$ & $35(8.9)$ & $80(20.3)$ & $202(51.1)$ & $211(53.4)$ \\
\hline Monthly or less & $87(17)$ & $10(11.5)$ & $4(4.6)$ & $14(16.1)$ & $47(54)$ & $53(60.9)$ \\
\hline 2-4 times a month & $24(4.7)$ & $2(8.3)$ & $1(4.2)$ & $3(12.5)$ & $11(45.8)$ & $15(62.5)$ \\
\hline 2-3 times a week & $5(1.0)$ & $2(40.0)$ & $1(20.0)$ & $2(40.0)$ & $2(40.0)$ & $3(60.0)$ \\
\hline \multicolumn{7}{|l|}{ Disclosure } \\
\hline Yes & $502(98.2)$ & 80 (15.9) & $40(8.0)$ & 96 (19.1) & $256(51.0)$ & $277(55.2)$ \\
\hline No & $9(1.8)$ & $5(55.6)$ & $1(11.1)$ & $3(33.3)$ & $6(66.7)$ & $5(55.6)$ \\
\hline
\end{tabular}

TVET, Technical and Vocational Education and Training

*Others: Seasonal and unemployed 
Table 2 Clinical characteristics and reported health problem of patients with HIV/AIDS, TASH, Ethiopia, 2019 ( $n=511)$

\begin{tabular}{|c|c|c|c|c|c|c|}
\hline \multirow[t]{2}{*}{ Patient characteristics } & \multirow{2}{*}{$\begin{array}{l}\text { Number } \\
\text { of participant } \\
\mathrm{n}(\%)\end{array}$} & \multicolumn{5}{|c|}{ Reported health problem in EQ-5D health dimensions } \\
\hline & & $\begin{array}{l}\text { Mobility } \\
\text { n (\%) }\end{array}$ & Self-care $\mathbf{n}(\%)$ & $\begin{array}{l}\text { Usual activity } \\
\text { n (\%) }\end{array}$ & $\begin{array}{l}\text { Pain/ } \\
\text { Discomfort } \\
\mathbf{n}(\%)\end{array}$ & $\begin{array}{l}\text { Anxiety/Depression } \\
\mathrm{n}(\%)\end{array}$ \\
\hline \multicolumn{7}{|l|}{ CD4 (Cells $\left./ \mathrm{mm}^{3}\right)$} \\
\hline$>500$ & $138(27.0)$ & $15(10.9)$ & $1(0.7)$ & $11(8.0)$ & $40(29.0)$ & $39(28.3)$ \\
\hline $350-500$ & $129(25.2)$ & $22(17.1)$ & $12(9.3)$ & $24(18.6)$ & $65(50.4)$ & $72(55.8)$ \\
\hline $200-350$ & $142(27.8)$ & $21(14.8)$ & $14(9.9)$ & $31(21.8)$ & $84(59.2)$ & $95(66.9)$ \\
\hline $100-200$ & $80(15.7)$ & $22(27.5)$ & $10(12.5)$ & $26(32.5)$ & $59(73.7)$ & $60(75.0)$ \\
\hline$<100$ & $22(4.3)$ & $5(22.7)$ & $4(18.2)$ & $7(31.8)$ & $14(63.6)$ & $16(72.7)$ \\
\hline \multicolumn{7}{|c|}{ WHO clinical stages (at diagnosis) } \\
\hline । & $67(13.3)$ & $16(23.9)$ & $3(4.5)$ & $10(14.9)$ & $40(59.7)$ & $42(62.7)$ \\
\hline$\|$ & $77(15.2)$ & $11(14.3)$ & $6(7.8)$ & $15(19.5)$ & $40(51.9)$ & $40(51.9)$ \\
\hline III & $190(37.6)$ & $28(14.7)$ & $16(8.4)$ & $32(16.8)$ & $83(43.7)$ & $95(50.0)$ \\
\hline IV & $171(33.9)$ & $27(15.8)$ & $14(8.2)$ & $40(23.4)$ & $97(56.7)$ & $102(59.6)$ \\
\hline \multicolumn{7}{|l|}{ Viral load (Copies/mL) } \\
\hline$<50$ copies $/ \mathrm{ml}$ & $440(86.1)$ & $70(15.9)$ & $33(7.5)$ & $81(18.4)$ & $217(49.3)$ & $236(53.6)$ \\
\hline $51-1000$ copies/ml & $17(3.3)$ & $3(17.6)$ & $2(11.8)$ & $2(11.8)$ & $9(52.9)$ & $10(58.8)$ \\
\hline > 1000 copies $/ \mathrm{ml}$ & $54(10.6)$ & $12(22.2)$ & $6(11.1)$ & $16(29.6)$ & $36(66.7)$ & $36(66.7)$ \\
\hline \multicolumn{7}{|l|}{ Treatment regimen } \\
\hline $\mathrm{AZT} / 3 \mathrm{TC} / \mathrm{EFV}$ & $30(5.9)$ & $5(16.7)$ & $1(3.3)$ & $4(13.3)$ & $10(33.30$ & $13(43.3)$ \\
\hline AZT/3TC/NVP & $28(5.5)$ & $4(14.3)$ & $1(3.6)$ & $3(10.7)$ & $14(50)$ & $13(46.4)$ \\
\hline TDF/3TC/EFV & $297(58.1)$ & $49(16.5)$ & $22(7.4)$ & $51(17.2)$ & $146(49.2)$ & $161(54.2)$ \\
\hline TDF/3TC/NVP & $37(7.2)$ & $4(10.8)$ & $4(10.8)$ & $10(27.0)$ & $19(51.4)$ & $19(51.4)$ \\
\hline$A B C+3 T C+E F V$ & $40(7.8)$ & $9(22.5)$ & $5(12.5)$ & $8(20.0)$ & $28(70.0)$ & $27(67.5)$ \\
\hline $\mathrm{TDF}+3 \mathrm{TC}+\mathrm{ATV} / \mathrm{r}$ & $41(8.0)$ & $7(10.8)$ & $3(7.3)$ & $12(29.3)$ & $27(65.9)$ & $29(70.7)$ \\
\hline Others* & $38(7.4)$ & $7(18.4)$ & $5(13.2)$ & $11(28.9)$ & $18(47.4)$ & $20(52.6)$ \\
\hline \multicolumn{7}{|l|}{ Number of medicine } \\
\hline 1 & $208(40.7)$ & $25(12)$ & $12(5.8)$ & $25(12)$ & $88(42.3)$ & $93(44.7)$ \\
\hline 2 & $150(29.4)$ & $26(17.3)$ & $11(7.3)$ & $31(20.7)$ & $84(56.0)$ & $90(60.0)$ \\
\hline 3 & $91(17.8)$ & $17(18.7)$ & $8(8.8)$ & $21(23.1)$ & 49 (53.8) & $53(58.2)$ \\
\hline 4 & $43(8.4)$ & $10(23.3)$ & $3(7)$ & $12(27.9)$ & $27(62.8)$ & $30(69.8)$ \\
\hline$>4$ & $19(3.7)$ & $7(36.8)$ & $7(36.8)$ & $10(52.6)$ & $14(73.7)$ & $16(84.2)$ \\
\hline \multicolumn{7}{|l|}{ Duration of treatment } \\
\hline$<1$ year & $4(0.8)$ & - & - & - & $4(100)$ & $1(25)$ \\
\hline $1-5$ years & $120(23.5)$ & $19(15.8)$ & $8(6.7)$ & $27(22.5)$ & $74(61.7)$ & 77 (64.2) \\
\hline$>5$ years & $386(75.7)$ & $66(17.1)$ & $33(8.5)$ & $72(18.7)$ & $183(47.4)$ & $203(52.6)$ \\
\hline \multicolumn{7}{|l|}{ Adherence } \\
\hline Good & $466(91.5)$ & $77(16.5)$ & $38(8.2)$ & $90(19.3)$ & $236(50.6)$ & $252(54.1)$ \\
\hline Fair & $13(2.6)$ & $3(13.1)$ & $1(7.7)$ & - & $7(53.8)$ & $9(69.2)$ \\
\hline Poor & $30(5.9)$ & $5(16.7)$ & $2(6.7)$ & $9(30)$ & $17(56.7)$ & $20(66.7)$ \\
\hline \multicolumn{7}{|l|}{ History of Rx failure } \\
\hline Yes & 94 (18.4) & $17(18.1)$ & $9(9.6)$ & $23(24.5)$ & $60(63.8)$ & $62(66)$ \\
\hline No & 417 (81.6) & $68(16.3)$ & $32(7.7)$ & $76(18.2)$ & $202(48.4)$ & $220(52.8)$ \\
\hline \multicolumn{7}{|l|}{ Comorbidities } \\
\hline No & $349(68.3)$ & 47 (13.5) & $21(6)$ & $53(15.2)$ & $156(44.7)$ & $175(50.1)$ \\
\hline Ols & $41(8)$ & $5(12.2)$ & $2(4.9)$ & $13(31.7)$ & $25(61)$ & $28(68.3)$ \\
\hline Non-Ols & $121(23.7)$ & $33(27.3)$ & $18(14.9)$ & $33(27.30$ & 81 (66.9) & 79 (65.3) \\
\hline
\end{tabular}

Rx, treatment; NVP, Nevirapine; $A B C$, Abacavir; Ols, Opportunistic infections ${ }^{*}$ Others: $A B C+3 T C+N V P, T D F+3 T C+L P V / r$ and $A Z T+3 T C+L P V / r$ 
$51.3 \%$ of participants reported at least slight problems on the anxiety/depression and pain/discomfort dimensions respectively (Table 3 ).

With respect to participants' health profile based on their clinical and socio-demographic characteristics, 163 (58.2\%) of female participants reported at least a slight problem on the pain/discomfort dimension. A nearly similar proportion of male $(112,55.4 \%)$ and female (170, $55 \%)$ participants reported at least a slight problem on the anxiety/depression dimension. Higher proportion of participants $(45,71.4 \%)$ from the younger age group (1829 years) reported having at least a slight problem on the anxiety/depression dimension. In contrast, 33 (71.7\%) of participants who were older than 60 years claimed having at least a slight problem on pain/discomfort dimension (Table 1). The number of participants with CD4 count of $>500$ cells $/ \mathrm{mm}^{3}$ who reported at least a slight problem on the anxiety/depression dimension was found to be 39 (28.3\%). In contrast, 60 (75\%) of participants with CD4 count of $100-200$ cells $/ \mathrm{mm}^{3}$ reported having a slight or more problems on the anxiety/depression dimension. Similarly, 16 (84.2\%) and 14 (73.7\%) of participants taking more than four medicines at a time reported a slight or more problems on the anxiety/depression and pain/discomfort dimensions, respectively (Table 2).

Utility values and factors influencing patients' utility values The overall median utility value of HIV/AIDS patients was found to be 0.94 (IQR $=0.87,1)$ and $80 \%$ (IQR $=70 \%$, 90\%) from the EQ-5D index and EQ-VAS result, respectively. The median utility values were found to be higher for participants in some socio-demographic and clinical characteristics categories. For instance, the median utility values were $0.96(\mathrm{IQR}=0.88,1)$ and $0.94(\mathrm{IQR}=0.77-$ $0.97)$ for participants in the age range of 30-39 years and those with a monthly household income of greater than 5000 ETB, respectively. There were significant differences in the EQ-5D utility index among different age groups, $p=0.004$, with a mean rank utility index of 232, $282,259,260$ and 192 for the age range of 18-29, 30-29, $40-49,50-59$ and $>60$, respectively. The difference in monthly household income showed significant difference in utility index, $p<0.001$, with mean rank utility value of 225 for $<1700$ ETB, 233 for 1700-5000 ETB and 299 for $>5000$ ETB. The Kruskal-Wallis test depicted that age, occupational status, and household monthly income significantly associated with participants' EQ-VAS scores with $p<0.001,0.008$ and 0.007 , respectively (Table 4).

Kruskal-Wallis analysis indicated that there was a statistically significant difference in EQ-5D utility values among patients with different CD4 count intervals, $p<0.001$. The number of medicines and comorbidities were significantly associated with EQ-5D utility index and EQ-VAS score, $p<0.001$. Participants with CD4 range of above 500 cells $/ \mathrm{mm}^{3}$ had higher (median $=1$; $\mathrm{IQR}=0.94,1) \mathrm{EQ}-5 \mathrm{D}$ utility value while those with CD4 range of below 100 cells $/ \mathrm{mm}^{3}$ had lower median value (0.88; IQR $=0.66,0.96)$. The median EQ-5D utility index value for patients who were receiving more than four medicines at a time was $0.89(\mathrm{IQR}=0.76,0.97)$ while patients on a single medicine had a median value of 0.96 $(\mathrm{IQR}=0.9,1)($ Table 5).

\section{Discussion}

This study provided critical information on HRQoL, health state utility values and factors that influence the values in the context of HIV/AIDS patients in Ethiopia. The study observed a higher frequency of health problems in all five dimensions of EQ-5D among HIV/AIDS patients compared to the general population of Ethiopia [23]. Consistent with previous studies from different parts of the world, HIV/AIDS patients had higher frequency of problems on the anxiety/depression dimension than the general population $[24,25]$. This indicates that patients with HIV/AIDS need better health care services than currently provided to reduce their health problems and improve quality of life with particular focus on mental and palliative care.

Based on respondents' descriptive profile, higher proportions $(45,71.4 \%)$ of the younger population claimed anxiety/depression problem than older age groups. This finding is consistent with a previous study that reported reduced depression symptoms with increase in age [26]. Depression has an impact beyond the reduction in

Table 3 Frequency of self-reported health profile of HIV/AIDS patients, TASH, Ethiopia, $2019(\mathbf{n}=511)$

\begin{tabular}{|c|c|c|c|c|c|}
\hline Health profile & Mobility n (\%) & Self-care n (\%) & $\begin{array}{l}\text { Usual activity } \\
\text { n (\%) }\end{array}$ & $\begin{array}{l}\text { Pain/Discomfort } \\
\text { n (\%) }\end{array}$ & $\begin{array}{l}\text { Anxiety/Depression } \\
\text { n (\%) }\end{array}$ \\
\hline No problem & $426(83.4)$ & $470(92)$ & $412(80.7)$ & $249(48.7)$ & $229(44.8)$ \\
\hline Slight problem & $48(9.4)$ & $33(6.4)$ & $71(13.9)$ & $143(28)$ & $151(29.5)$ \\
\hline Moderate problem & $22(4.3)$ & $4(0.8)$ & $22(4.2)$ & $87(17)$ & $103(20.2)$ \\
\hline Severe problem & $15(2.9)$ & $4(0.8)$ & $6(1.2)$ & $32(6.3)$ & $27(5.3)$ \\
\hline Extreme problem & 0 & 0 & 0 & 0 & $1(0.2)$ \\
\hline
\end{tabular}


Table 4 Socio-demographic factor associated with EQ-5D and EQ-VAS score of patients with HIV/AIDS, TASH, Ethiopia, $2019(n=511)$

\begin{tabular}{|c|c|c|c|c|c|c|}
\hline \multirow[t]{2}{*}{ Patient characteristics } & \multicolumn{3}{|l|}{ EQ-5D } & \multicolumn{3}{|l|}{ EQ-VAS } \\
\hline & Median [IQR] & Mean rank & $p$ value & Median [IQR] & Mean rank & $p$ value \\
\hline \multicolumn{7}{|l|}{ Gender } \\
\hline Male & $0.96[0.86-1]$ & 261 & 0.54 & $85[70,90]$ & 252 & 0.65 \\
\hline Female & $0.94[0.87-1]$ & 253 & & 80 [70-95] & 258 & \\
\hline \multicolumn{7}{|l|}{ Age } \\
\hline $18-29$ & $0.94[0.84-0.97]$ & 232 & $0.004^{*}$ & 80 [60-90] & 217 & $<0.001^{*}$ \\
\hline $30-39$ & $0.96[0.88-1]$ & 282 & & 85 [70-95] & 284 & \\
\hline $40-49$ & $0.94[0.88-1]$ & 259 & & 85 [70-95] & 271 & \\
\hline $50-59$ & $0.94[0.86-1]$ & 260 & & 80 [70-90] & 250 & \\
\hline$>60$ & $0.89[0.75-0.97]$ & 192 & & $65[50-85.5]$ & 177 & \\
\hline \multicolumn{7}{|l|}{ Marital status } \\
\hline Unmarried & $0.96[0.85-1]$ & 253 & 0.11 & 80 [70-95] & 251 & 0.26 \\
\hline Married & $0.96[0.88-1]$ & 268 & & 85 [70-95] & 268 & \\
\hline Divorced & $0.94[0.88-0.97]$ & 250 & & 80 [65-90] & 243 & \\
\hline Widowed & $0.92[0.86-0.97]$ & 216 & & 80 [60-90] & 224 & \\
\hline \multicolumn{7}{|l|}{ Education } \\
\hline No formal education & $0.94[0.86-0.97]$ & 233 & 0.27 & 80 [65-90] & 234 & 0.24 \\
\hline Grade(1-4) & $0.94[0.86-1]$ & 255 & & 80 [64.75-91.25] & 245 & \\
\hline Grade (5-8) & $0.94[0.88-1]$ & 250 & & 80 [70-90] & 246 & \\
\hline High school & $0.94[0.86-1]$ & 252 & & 80 [70-95] & 262 & \\
\hline TVET/diploma & $0.96[0.88-1]$ & 276 & & 85 [70-95] & 280 & \\
\hline 1st degree and above & $0.97[0.89-1]$ & 301 & & 90 [78.75-95] & 293 & \\
\hline \multicolumn{7}{|l|}{ Occupational status } \\
\hline Employed & $0.97[0.88-1]$ & 280 & $0.001^{*}$ & 85 [75-93.75] & 280 & $0.008^{*}$ \\
\hline Private business & $0.94[0.88-1]$ & 261 & & 85 [70-90] & 255 & \\
\hline Pensioner & 0.85 [0.83-93] & 146 & & $70[45-80]$ & 261 & \\
\hline Student & $0.94[0.87-0.97]$ & 228 & & 80 [60-85] & 194 & \\
\hline Housewife & 094 [0.88-1] & 258 & & 80 [70-95] & 267 & \\
\hline Others** & $0.86[0.66-0.94]$ & 164 & & 60 [50-95] & 196 & \\
\hline \multicolumn{7}{|l|}{ Monthly income } \\
\hline$<1700$ ETB & $0.94[0.85-0.99]$ & 225 & $0.001^{*}$ & $80[65-90]$ & 218 & $0.007^{*}$ \\
\hline 1700-5000 ETB & $0.94[0.87-0.97]$ & 233 & & 80 [70-90] & 241 & \\
\hline$>5000$ ETB & $0.97[0.77-0.97]$ & 299 & & 90 [80-96.5] & 293 & \\
\hline \multicolumn{7}{|l|}{ Smoking status } \\
\hline Daily & 0.99 & 388 & 0.27 & - & 293 & 0.49 \\
\hline Occasionally & $0.9[0.71-1]$ & 215 & & 77.5 [55-92.5] & 210 & \\
\hline Not at all & $0.94[0.87-0.94]$ & 257 & & 80 [70-90] & 257 & \\
\hline \multicolumn{7}{|l|}{ Alcohol consumption } \\
\hline Never & $0.94[0.88-1]$ & 258 & 0.77 & 80 [70-95] & 260 & 0.63 \\
\hline Monthly or less & $0.94[0.86-0.97]$ & 242 & & 77.5 [70-90] & 237 & \\
\hline 2-4 times a month & $0.97[0.8-1]$ & 268 & & 85 [70-90] & 256 & \\
\hline 2-3 times a week & $0.97[0.88-1]$ & 275 & & 75 & 271 & \\
\hline \multicolumn{7}{|l|}{ Disclosure status } \\
\hline Yes & $0.94[0.87-1]$ & 257 & 0.09 & 80 [70-90] & 257 & 0.35 \\
\hline No & $0.88[0.67-1]$ & 175 & & 75 [70-90] & 211 & \\
\hline
\end{tabular}

TVET, Technical and Vocational Education and Training

**Others: Seasonal and unemployed 
Table 5 Clinical characteristics associated with EQ-5D and EQ-VAS score of patients with HIV/AIDS, TASH, Ethiopia, 2019 $(\mathrm{n}=\mathbf{5 1 1})$

\begin{tabular}{|c|c|c|c|c|c|c|}
\hline \multirow[t]{2}{*}{ Patient character } & \multicolumn{3}{|l|}{ EQ-5D } & \multicolumn{3}{|l|}{ EQ-VAS } \\
\hline & Median [IQR] & Mean rank & $p$ value & Median [IQR] & Mean rank & $p$ value \\
\hline \multicolumn{7}{|l|}{ CD4 cells $/ \mathrm{mm}^{3}$} \\
\hline$>500$ & $1[0.94-1]$ & 339 & \multirow[t]{5}{*}{$<0.001^{*}$} & 90 [82-99.75] & 346 & \multirow[t]{5}{*}{$<0.001^{*}$} \\
\hline$[350-500]$ & $0.94[0.88-1]$ & 258 & & $85[70.25-90]$ & 268 & \\
\hline [200-350] & $0.94[0.86-0.97]$ & 232 & & $77.5[65-85]$ & 213 & \\
\hline [100-200] & $0.88[0.76-0.95]$ & 172 & & 70 [60-85] & 184 & \\
\hline$<100$ & $0.88[0.66-0.96]$ & 184 & & $60[50-83.75]$ & 166 & \\
\hline \multicolumn{7}{|c|}{ WHO clinical stage at diagnosis } \\
\hline 1 & $0.94[0.85-0.97]$ & 233 & \multirow[t]{4}{*}{0.06} & $85[72.5-90]$ & 264 & \multirow[t]{4}{*}{0.08} \\
\hline$\|$ & $0.96[0.87-1]$ & 261 & & 85 [70-95] & 266 & \\
\hline III & $0.96[0.89-1]$ & 273 & & 82.5 [70-95] & 265 & \\
\hline IV & $0.94[0.86-1]$ & 235 & & 80 [65-90] & 229 & \\
\hline \multicolumn{7}{|l|}{ Viral load } \\
\hline$<50$ copies $/ \mathrm{ml}$ & $0.95[0.88-1]$ & 263 & \multirow[t]{3}{*}{$0.005^{*}$} & 85 [70-95] & 267 & \multirow[t]{3}{*}{$<0.001^{*}$} \\
\hline 51-1000 copies/ml & $0.94[0.88-1]$ & 257 & & 82.5 [70-95] & 260 & \\
\hline$>1000$ copies/ml & $0.88[0.7-0.98]$ & 196 & & $70[60-80]$ & 169 & \\
\hline \multicolumn{7}{|l|}{ Type of medicine } \\
\hline AZT/3TC/EFV & $0.97[0.94-1]$ & 309 & \multirow[t]{7}{*}{$0.004^{*}$} & 82.5 [70-91.75] & 286 & \multirow[t]{7}{*}{$0.002^{*}$} \\
\hline AZT/3TC/NVP & $0.96[0.88-1]$ & 271 & & 85 [71.25-90] & 278 & \\
\hline TDF/3TC/EFV & $0.96[0.88-1]$ & 264 & & 85 [70-95] & 268 & \\
\hline TDF/3TC/NVP & $0.94[0.89-1]$ & 269 & & 85 [75-95] & 280 & \\
\hline$A B C+3 T C+E F V$ & $0.88[0.82-0.96]$ & 189 & & 70 [60-80] & 192 & \\
\hline$T D F+3 T C+A T V / r$ & $0.82[0.77-0.97]$ & 253 & & 70 [51.25-85] & 238 & \\
\hline Others ${ }^{* *}$ & $0.94[0.86-1]$ & 205 & & 80 [65-90] & 193 & \\
\hline \multicolumn{7}{|l|}{ Number of medicine } \\
\hline 1 & $0.96[0.9-1]$ & 289 & \multirow[t]{5}{*}{$<0.001^{*}$} & 90 [70-95] & 289 & \multirow[t]{5}{*}{$<0.001^{*}$} \\
\hline 2 & $0.94[0.85-0.97]$ & 240 & & 80 [70-90] & 246 & \\
\hline 3 & $0.94[0.86-1]$ & 239 & & 80 [65-90] & 240 & \\
\hline 4 & $0.91[0.83-0.97]$ & 225 & & 75 [60-90] & 214 & \\
\hline$>4$ & $0.89[0.76-0.97]$ & 179 & & $70[50-80]$ & 144 & \\
\hline \multicolumn{7}{|l|}{ Duration of treatment } \\
\hline$<1$ year & $0.96[0.94-0.96]$ & 49 & \multirow[t]{3}{*}{0.08} & 85 [72.5-90] & 51 & \multirow[t]{3}{*}{0.12} \\
\hline $1-5$ years & $0.92[0.86-0.97]$ & 50 & & 80 [66.25-90] & 45 & \\
\hline$>5$ years & $0.96[0.88-1]$ & 51 & & 82.5 [70-95] & 55 & \\
\hline \multicolumn{7}{|l|}{ Adherence } \\
\hline Good & $0.95[0.88-1]$ & 259 & \multirow[t]{3}{*}{0.14} & 80 [70-90] & 259 & 0.08 \\
\hline Fair & $0.93[0.84-1]$ & 225 & & 72.5 [56-93.75] & 223 & \\
\hline Poor & $0.89[0.7-1]$ & 209 & & $70[60-90]$ & 201 & \\
\hline History of treatment $f$ & & & & & & \\
\hline Yes & $0.92[0.83-0.97]$ & 210 & $0.001^{*}$ & 70 [60-85] & 195 & $<0.001^{*}$ \\
\hline No & $0.96[0.88-1]$ & 266 & & 85 [70-95] & 270 & \\
\hline Comorbidities & & & & & & \\
\hline No & $0.96[0.88-1]$ & 216 & $0.001^{*}$ & 85 [70-95] & 213 & $<0.001^{*}$ \\
\hline Ols & $0.92[0.84-0.97]$ & 273 & & 70 [55-90] & 274 & \\
\hline Non-Ols & 0.92 [0.8-0.97] & 336 & & 80 [65-90] & 237 & \\
\hline
\end{tabular}

*Significant value

**Others: ABC + 3TC + NVP, TDF + 3TC + LPV/r and AZT + 3TC + LPV/r; NVP: Nevirapine; ABC: Abacavir; Ols: Opportunistic infect 
quality of life and it is significantly associated with a fall in the level of treatment adherence [27]. Consequently, the younger population needs more psychological support including HIV-specialist counselling, psychotherapies and mental health intervention to cope with anxiety and depression. These interventions are expected to improve the patient quality of life and to enhance treatment outcomes through improved adherence to the treatment regimen $[27,28]$. Almost three-fourth of participants' older than 60 years of age reported problems on the pain/discomfort dimension. This could be associated with age and comorbidities, showing the importance of managing comorbidities and provision of palliative care for older HIV/AIDS patients [29].

In the current study, the overall median EQ-5D utility index and EQ-VAS score were reported as 0.94 $(\mathrm{IQR}=0.87,1)$ and $80 \%(\mathrm{IQR}=70,90)$, respectively. The EQ-5D index recorded from this study were higher than the finding reported from Vietnam $($ mean $=0.65$; $\mathrm{SD}=0.27)$ and Colombia (mean $=0.85 ; \mathrm{SD}=0.21)[24$, 30]. The utility value reported from the patients or society is country specific and it can be affected by cultural belief. As a result, such discrepancies usually occur in the utility scores reported from different countries [31]. Since utility is affected by socio-cultural vaue of the society, the higher utility report of the patients in our study may not reflect the better performance of the health care service in Ethiopia. The EQ-VAS scores were recorded as lower than the general population that reported the median value of $90 \%(\mathrm{IQR}=20)$ and this could be acceptable since several studies indicated that HIV/AIDS patients have reduced quality of life in comparison to general population [19, 23, 32, 33]. Findings from South Africa and Colombia depicted better EQ-VAS scores in comparison to our study [30,34]. If we consider the better economic status of South Africa and Colombia the reported utility value from Ethiopian patient seems worthy. But, further work has to be done to improve the HRQoL of the patients with the fact that reported HRQoL of the patients is lower than the general population.

This study examined the difference in utility value of patients based on demographic and clinical characteristics. Patients with low income demonstrated a lower utility value in comparison to the higher-income patient population. Studies showed that HIV/AIDS patients and other chronic diseases showed better HRQoL with improved economic status [24, 35]. HIV/AIDS also affects patients' economic status keeping individuals and families in the poverty trap [36]. Improved health outcome needs health care services and economic intervention, therefore, providing social and financial support for low-income HIV/AIDS patients should be considered. From our study, it was found that age, occupational status, and household monthly income were significantly associated with the EQ-5D index and EQ-VAS score. This revealed the convergent validity of EQ-5D-5L and EQVAS as depicted in a previous study from Vietnam [24].

Participants with a CD4 range of above 500 reported higher utility scores while those with a CD4 range of below 200 claimed lower utility scores. This result is consistent with studies that reported better quality of life with increased CD4 [37, 38]. In addition to the CD4 range, the number of medicines and comorbidities were significantly associated with utility scores. Viral load and history of treatment failure were also found to be associated with EQ-5D utility index and EQ-VAS score of the patient. Existing literature showed that change in medicine and an increase in viral load may cause psychological distress on patients and this would affect the utility score [39]. Therefore, counseling patients on treatment adherence to prevent treatment failure and promoting health education for reducing the magnitude of comorbidities may be helpful in improving their HRQoL.

As a limitation, the study conducted at single hospital and participants for the study were recruited using convenience sampling approach and this may lead to sampling biases. These issues might have influenced the generalizability of the findings. This study has several stregths and contribution to the current literature. To the best of the authors' knowledge, this study is the first of its kind to establish the utility value for patients with different health states of HIV/AIDS in the Ethiopian setting. The utility value reported from the current study can be used as an input for conducting future economic evaluations of different HIV/AIDS interventions. The study enrolled large sample size and this increases the precision of the finding.

\section{Conclusion}

This study found that anxiety/depression and pain/discomfort were more frequently reported health problems among HIV/AIDS patients. The higher frequency of problems in each health dimensions and lower overall utility values were recorded from HIV/AIDS patients in comparison to the general population. The utility value of the patients was significantly associated with age, income, $\mathrm{CD} 4$, the number of medicine the patient was taking at a time and comorbidities. Service providers should work to reduce problems of anxiety/depression and pain/discomfort to further improve patients' overall utility values.

\section{Abbreviations}

ART: Antiretroviral Treatment; CD4: Cluster of differentiation 4; EQ-5D-5L: EuroQol-5 Dimensions-5 Levels; EQ-VAS: EuroQol-Visual Analog Scales; HIV/AIDS: Human Immunodeficiency Virus/Acquired Immune-Deficiency Syndrome: HRQoL: Health-related Quality of Life; IQR: Interquartile Range; TASH: Tikur Anbessa Specialized Hospital. 


\section{Acknowledgements}

We would like to extend our gratitude to Addis Ababa University for funding this research work. Our deepest thanks also go to participants of the study for their time and the valuable information provided.

\section{Authors' contributions}

YB and GBG conceived the project. YB, BS, EEA, and GBG designed the methods and prepared the data collection tools. YB carried out data collection and verified the collected data. YB, EEA, BS and GBG performed data analysis. $Y B$ wrote the draft manuscript and all authors reviewed the manuscript and provided critical scholarly feedback. All authors read and approved the final manuscript.

\section{Funding}

This project was supported by Addis Ababa University and in part, by a Canada Research Chair in Economics of Infectious Diseases held by Beate Sander (CRC-950-232429).

\section{Availability of data and materials}

The datasets used during the current study are available from the corresponding author on reasonable request.

\section{Ethics approval and consent to participate}

Ethical clearance was obtained from the Ethics Review Board of the School of Pharmacy (Ref.No: ERB/SOP/53/03/2019). Permission was also sought from the study hospital and participants were asked for verbal consent before an interview. Patient privacy and confidentiality were secured by interviewing the participant in a separate place from the waiting area. Access of data was limited only for the research team.

\section{Consent for publication}

Not applicable.

\section{Competing interests}

The project was designed and conducted without any interference from funding organization and the authors declare that they have no competing interests.

\section{Author details}

1 School of Pharmacy, College of Health Sciences, Mekelle University, Mek'ele, Ethiopia. ${ }^{2}$ Department of Pharmaceutics and Social Pharmacy, School of Pharmacy, College of Health Sciences, Addis Ababa University, Addis Ababa, Ethiopia. ${ }^{3}$ Toronto Health Economics and Technology Assessment (THETA) Collaborative, University Health Network, Toronto, ON, Canada. ${ }^{4}$ Institute of Health Policy, Management and Evaluation (IHPME), University of Toronto, Toronto, ON, Canada. ${ }^{5}$ Public Health Ontario, Toronto, ON, Canada. ${ }^{6}$ ICES, Toronto, ON, Canada.

Received: 12 May 2020 Accepted: 6 January 2021

Published online: 19 January 2021

\section{References}

1. Frank TD, Carter A, Jahagirdar D, Biehl MH, Douwes-Schultz D, Larson SL, Arora M, Dwyer-Lindgren L, Steuben KM, Abbastabar H, Abu-Raddad LJ. Global, regional, and national incidence, prevalence, and mortality of HIV, 1980-2017, and forecasts to 2030, for 195 countries and territories: a systematic analysis for the Global Burden of Diseases, Injuries, and Risk Factors Study 2017. Lancet HIV. 2019;6:12.

2. United Nations Programme on HIV/AIDS (UNAIDS). Global HIV and AIDS statistics 2019 fact sheet. 2019. https://www.unaids.org/en/resources/ fact-sheet. Accessed 11 Nov 2019

3. United Nations Programme on HIV/AIDS (UNAID). Fast-track: ending the AIDS epidemic by 2030. 2014. https://www.unaids.org/en/resources/ documents/2014/JC2686WAD2014 report. Accessed 11 Nov 2019.

4. Centers for Disease Control and Prevention (CDC). Ethiopia Country Profile. 2019. https://www.cdc.gov/globalhivtb/where-we-work/ethiopia/ ethiopia.html. Accessed 11 Nov 2019.
5. Federal Ministry of Health (FMoH). Fifth health account FMOH, Ethiopia. 2014. https://www.hfgproject.org/wp-content/uploads/2014/04/Ethio pia-NHA-Findings-Briefing-Notes.pdf. Accessed 17 Nov 2019.

6. Arts EJ, Hazuda DJ. HIV-1 antiretroviral drug therapy. Cold Spring Harb Perspect Med. 2012;2:4.

7. World Health Organization (WHO). Consolidated guidelines on the use of antiretroviral drugs for treating and preventing HIV infection. 2016. https ://www.who.int/hiv/pub/arv/arv-2016/en/. Accessed 11 Nov 2019.

8. European Network for Health Technology Assessment (Eunethta). Eunethta, 2015. Endpoints used for Relative Effectiveness Assessment: Health-related quality of life and utility measures. 2015. https://eunet hta.eu/wp-content/uploads/2018/01/Endpoints-used-for-Relative-Effec tiveness-Assessment-Health-related-quality-of-life-and-utility-measu res_Amended-JA1-Guideline_Final-Nov-2015.pdf. Accessed 11 Nov 2019.

9. Whitehead SJ, Ali S. Health outcomes in economic evaluation: the QALY and utilities. Br Med Bull. 2010;96(1):5-21.

10. World Health Organization (WHO). HOQOL-HIV Instrument.2002. https:// www.who.int/mental_health/media/en/613.pdf. Accessed 11 Nov 2019.

11. Janssen MF, Pickard AS, Golicki D, Gudex C, Niewada M, Scalone L, et al. Measurement properties of the EQ-5D-5L compared to the EQ-5D-3L across eight patient groups: a multi-country study. Qual Life Res. 2013:22:1717-27.

12. World Health Organization (WHO). Measuring quality of life. 1995. https:// www.who.int/healthinfo/survey/whoqolqualityoflife/. Accessed 11 Nov 2019.

13. Gusi N, Olivares PR, Rajendram R. The EQ-5D health-related quality of life questionnaire. Handbook of disease burdens and quality of life measures. New York: Springer; 2010. p. 87-99.

14. Alemu A, Yenealem A, Feleke A, Meseret S. Health related quality of life assessment and associated factors among people on highly active antiretroviral therapy at Felege Hiwot referral hospital, Bahir Dar, north West Ethiopia. J AIDS Clin Res. 2013;5:2-6.

15. Devlin NJ, Brooks R. EQ-5D and the EuroQol group: past, present and future. Appl Health Econ Health Pol. 2017;15:127-37.

16. Despiégel N, Anger D, Martin M, Monga N, Cui Q, Rocchi A, et al. Costeffectiveness of dolutegravir in HIV-1 treatment-naive and treatmentexperienced patients in Canada. Infect Dis Ther. 2015;4:337-53.

17. Alemayehu M, Wubshet M, Mesfin N, Tamiru A, Gebayehu A. Healthrelated quality of life of HIV infected adults with and without Visceral Leishmaniasis in Northwest Ethiopia. Health Qual Life Outcomes. 2017;15:65.

18. Emamu S, Sebsebe F, Bedemariam G. (PDF) Quality of life of HIV/AIDS patients on highly active antiretroviral therapy at Tikur Anbessa Specialized Hospital, Addis Ababa, Ethiopia: a crosssectional study. 2017. https:// www.researchgate.net/publication/328676130. Accessed 11 Nov 2019.

19. Surur AS, Teni FS, Wale W, Ayalew Y, Tesfaye B. Health related quality of life of HIV/AIDS patients on highly active anti-retroviral therapy at a university referral hospital in Ethiopia. BMC Health Serv Res. 2017;17:737.

20. Deribew A, Tesfaye M, Hailmichael Y, Negussu N, Daba S, Wogi A, et al. Tuberculosis and HIV co-infection: its impact on quality of life. Health Qual Life Outcomes. 2009;7:105.

21. Charan J, Biswas T. How to calculate sample size for different study designs in medical research? Indian J Psychol Med. 2013;35(2):121.

22. EuroQol Research Foundation. EQ-5D-5L User Guide. 2019. https://euroq ol.org/publications/user-guides. Accessed 19 Jul 2019

23. Welie AG, Gebretekle GB, Stolk E, Mukuria C, Krahn MD, Enquoselassie F, et al. Valuing health state: an EQ-5D-5L value set for Ethiopians. Value Health Reg Issues. 2020;22:7-14.

24. Tran BX, Ohinmaa A, Nguyen LT. Quality of life profile and psychometric properties of the EQ-5D-5L in HIV/AIDS patients. Health Qual Life Outcomes. 2012;10:132.

25. Miners A, Phillips A, Kreif N, Rodger A, Speakman A, Fisher M, et al. Health-related quality-of-life of people with HIV in the era of combination antiretroviral treatment: a cross-sectional comparison with the general population. Lancet HIV. 2014;1:32-40.

26. McGowan JA, Sherr L, Rodger AJ, Fisher M, Miners A, Anderson J, et al. Age, time living with diagnosed HIV infection, and self-rated health. HIV Med. 2017:18:89-103. 
27. Jun TAO, Han-Zhu Q, Aaron M, Yuhua R, Bryan ES. Effects of depression and anxiety on antiretroviral therapy adherence among newly diagnosed HIV-infected Chinese men who have sex with men. AIDS. 2017;31:401.

28. British Psychological Society, British HIV Association, Medical Foundation for AIDS and Sexual Health. Standards for psychological support for adults living with HIV. 2011.

29. Higginson I. Palliative care: a review of past changes and future trends. J Public Health (Oxf). 1993;15:3-8.

30. Keaei M, Kuhlmann J, Conde R, Evers SM, Gonzalez J, Govers M, et al. Health-related quality of life of patients with HIV/AIDS in Bogota. Colombia Value Health Reg Issues. 2016;11:68-72.

31. Mahlich J, Dilokthornsakul P, Sruamsiri R, Chaiyakunapruk N. Cultural beliefs, utility values, and health technology assessment. CERA. 2018;16:19.

32. Beard J, Feeley F, Rosen S. Economic and quality of life outcomes of antiretroviral therapy for HIV/AIDS in developing countries: a systematic literature review. AIDS Care. 2009;21:1343-56.

33. Harding $R$, Simms V, Penfold S, Downing J, Namisango E, Powell RA, et al. Quality of life and wellbeing among HIV outpatients in East Africa: a multicentre observational study. BMC Infect Dis. 2014;14:613.

34. Nglazi MD, West SJ, Dave JA, Levitt NS, Lambert EV. Quality of life in individuals living with HIV/AIDS attending a public sector antiretroviral service in Cape Town. South Africa BMC Public Health. 2014;14:676.
35. Lemos CF, Rodrigues MP, Veiga JRP. Family income is associated with quality of life in patients with chronic kidney disease in the pre-dialysis phase: a cross sectional study. Health Qual Life Outcomes. 2015;13:202.

36. Boutayeb A. The impact of HIV/AIDS on human development in African countries. BMC Public Health. 2009;9:S3. https://doi. org/10.1186/1471-2458-9-S1-S3

37. Karkashadze E, Gates MA, Chkhartishvili N, DeHovitz J, Tsertsvadze T. Assessment of quality of life in people living with HIV in Georgia. Int J STD AIDS. 2017;28:672-8.

38. Liping M, Peng X, Haijiang L, Lahong J, Fan L. Quality of life of people living with HIV/AIDS: a cross-sectional study in Zhejiang Province. China PLOS ONE. 2015;10:8.

39. Weinstein TL, Li X. The relationship between stress and clinical outcomes for persons living with HIV/AIDS: a systematic review of the global literature. AIDS Care. 2016;28:160-9.

\section{Publisher's Note}

Springer Nature remains neutral with regard to jurisdictional claims in published maps and institutional affiliations.
Ready to submit your research? Choose BMC and benefit from:

- fast, convenient online submission

- thorough peer review by experienced researchers in your field

- rapid publication on acceptance

- support for research data, including large and complex data types

- gold Open Access which fosters wider collaboration and increased citations

- maximum visibility for your research: over $100 \mathrm{M}$ website views per year

At $\mathrm{BMC}$, research is always in progress.

Learn more biomedcentral.com/submissions 